\title{
Rol de infliximab en enfermedad inflamatoria intestinal. Experiencia local
}

\author{
DANIELA SIMIAN ${ }^{1, a}$, MARÍA ISABEL QUIJADA ${ }^{1}$, \\ JAIME LUBASCHER ${ }^{2}$, RAÚL ACUÑA ${ }^{2}$, RODRIGO QUERA ${ }^{2}$
}

'Dirección Académica, Clínica Las Condes. ${ }^{2}$ Departamento de

Gastroenterología, Clínica Las Condes, Santiago, Chile. aEnfermera.

Recibido el 3 de diciembre de 2012, aceptado el 19 de junio de 2013.

Correspondencia a: Dr. Rodrigo Quera Lo Fontecilla 441, Las Condes. Teléfono: 82894787 E-mail:rquera@clc.cl

\section{Treatment of inflammatory bowel disease with infliximab. Experience in 25 patients}

Background: Biological therapy has an important role in the treatment of Inflammatory Bowel Disease (IBD). However, the use of these drugs is resisted due to fears about their side effects. Aim: To report the experience with the use of Infliximab in patients with IBD. Material and Methods: Descriptive study of a historical cohort of patients with IBD treated between 2007 and 2012 with Infliximab. A favorable clinical response was considered when general, intestinal and extra-intestinal symptoms subsided after the second or third dose of the drug. Endoscopic or imaging response was evaluated between three and six months of treatment. Results: Twenty five patients aged 18 to 61 years (12 women) were included. Sixteen had Cohn's Disease and 9 had Ulcerative Colitis. Treatment was indicated due to refractory disease in 13 patients, perianal involvement in nine, stenosis in two and pyoderma gangrenosum in one. Ten patients initiated Infliximab within less than two years of diagnosis. Twenty-two patients received combined treatment with immunosuppressive medications and the other three patients were treated exclusively with Infliximab. A favorable clinical response was observed in $88 \%$ after the second dose and $64 \%$ had endoscopic or imaging remission after 3-6 months. Twelve patients discontinued Infliximab, due to bad response to treatment in three patients, economic cost in three patients, and patient/doctor decision in six. Only three patients had side effects (herpes zoster and sinusitis). None of these motivated the discontinuation of treatment. Conclusions: In this cohort of patients with IBD, the use of Infliximab was associated with endoscopic or imaging remission in 64\% of cases after 3-6 months of treatment with no major side effects.

(Rev Med Chile 2013; 141: 1158-1165).

Key words: Antibodies, monoclonal; Colitis, ulcerative; Crohn disease; Infliximab.
L a enfermedad inflamatoria intestinal (EII) es una enfermedad crónica idiopática en la que existe una inflamación de la mucosa intestinal. Esta entidad incluye la enfermedad de Crohn (EC), la colitis ulcerosa (CU) y la EII no clasificable. La EII tiende a presentar un curso crónico con períodos de remisión y actividad de duración variable, lo que hace que su tratamiento sea complejo. Los objetivos del tratamiento son lograr remisión sin esteroides, inducir y mantener la mucosa sana, remisión radiológica, mantención de la función gastrointestinal normal y prevenir cirugías, hospitalizaciones y discapacidad, todo lo cual se traduce en una mejor calidad de vida para el paciente ${ }^{1-4}$.

Actualmente, su tratamiento es sólo sintomático a través del uso de moduladores de la inflamación, como mesalazina o ácido 5-aminosalicílico, esteroides e inmunosupresores ${ }^{5-7}$. La terapia convencional logra controlar la inflamación, pero aparentemente no cambia el curso de la enfermedad. Más aun, muchos pacientes son refractarios 
a dichas terapias ${ }^{8}$. Aunque los esteroides son efectivos para inducir la remisión, ellos fracasan en mantener esta condición, así como en lograr la mejoría endoscópica ${ }^{9}$. La terapia biológica juega un rol fundamental en el control de los factores proinflamatorios, logrando obtener la remisión de manera rápida y efectiva, y la mantención de ella en el tiempo.

En la actualidad cuatro fármacos biológicos anti-factor necrosis tumoral (anti-TNF $\alpha$ ) han sido aprobados para su uso en EII: infliximab, adalimumab, certolizumab y golimumab ${ }^{9,10}$. Infliximab es un anticuerpo monoclonal quimérico dirigido contra el factor de necrosis tumoral TNF- $\alpha$, el cual actúa uniéndose a la porción TNF unida a membrana y circulante, lo que produce una reacción de citotoxicidad mediada por células y un aumento de la muerte celular activada por linfocitos $T^{11,12}$.

Diferentes estudios han demostrado la eficacia de la terapia con infliximab tanto en EC como en $\mathrm{CU}$, logrando cumplir los objetivos del tratamiento en estas patologías 1,3,4,10,12-14,15. Infliximab está indicado en pacientes con corticodependencia, refractariedad a corticoides e inmunomoduladores, fístula perianal y manifestaciones extraintestinales asociadas a EII ${ }^{9,12-14,16}$. No obstante, aún existe una subutilización de esta terapia por temor a los eventos adversos asociados a su uso y por los costos del tratamiento ${ }^{2,17}$.

El objetivo de este trabajo es describir el uso de infliximab en pacientes con EII tratados en Clínica Las Condes (CLC) durante los años 20072012, cuantificando su respuesta al tratamiento, la presencia de eventos adversos y la evolución en el tiempo.

\section{Material y Método}

Estudio observacional descriptivo de una cohorte histórica de pacientes con diagnóstico de EII tratados con infliximab entre los años 2007 y 2012 en CLC.

Criterios de inclusión: pacientes que pertenecían a la cohorte histórica de $\mathrm{EII}^{18,19}$, en tratamiento con terapia biológica, $\geq 18$ años, con seguimiento mínimo de tres meses desde el inicio del tratamiento. Se excluyó a los pacientes que recibieron terapia biológica en otra institución. El registro de pacientes fue aprobado por el Comité de Ética de CLC. Todos los pacientes fueron presentados en una reunión interdisciplinaria de patología inflamatoria intestinal, para decidir la indicación de la terapia con infliximab.

Se incluyeron las características demográficas, clínicas, de tratamiento y eventos adversos asociadas al uso del medicamento.

Todos los pacientes fueron tratados con infliximab, fármaco aprobado por la US Food and Drug Administration en EII para el tratamiento de la CU y EC moderada a severa y EC fistulizante. Se administró infliximab $5 \mathrm{mg} / \mathrm{kg}$ endovenoso en las semanas 0,2 y 6 como fase de inducción, seguido por una terapia de mantención cada 8 semanas ${ }^{5-7,9}$.

Se consideró respuesta clínica favorable a la regresión de los síntomas generales, digestivos y extraintestinales posterior a la $2^{\text {a }} \mathrm{o} 3^{\text {a }}$ dosis según la indicación de la terapia. Se evaluó la respuesta endoscópica y/o radiológica con colonoscopia o resonancia magnética (RM) de pelvis según corresponda, a los 3 a 6 meses de iniciado el tratamiento. En pacientes con CU se consideró remisión endoscópica la ausencia de inflamación de la mucosa (puntaje 0-1) según la Clasificación de Mayo $^{20}$. En pacientes con EC y compromiso inflamatorio se consideró remisión endoscópica de la mucosa a un puntaje $\leq 2$, según el score endoscópico SES-CD ${ }^{21,22}$. En pacientes con EC y compromiso perianal se consideró una buena respuesta radiológica la reducción de la fístula a lo menos en $50 \%$ en el control por RM de pelvis ${ }^{23}$.

En congruencia con el objetivo del estudio se realizó estadística descriptiva para reportar los resultados de las variables. Se utilizó el programa estadístico Stata 12. La única variable continua fue la edad, la cual se describió a través de la mediana y rango, porque no tenía distribución normal. El resto fueron variables categóricas, las que se describieron a través de la frecuencia absoluta y relativa $(\%)$.

\section{Resultados}

De un total de 217 pacientes evaluados en CLC durante el período 2007-2012, 56 de ellos con EC, 155 con diagnóstico de CU y 6 con EII no clasificable, se incluyeron 25 pacientes tratados con infliximab en CLC (Figura 1). Dieciséis tenían el diagnóstico de EC y nueve de CU, se distribuyeron por igual en ambos sexos. La mediana de edad fue 36 años (18-61 años). Se clasificó a los pacientes 


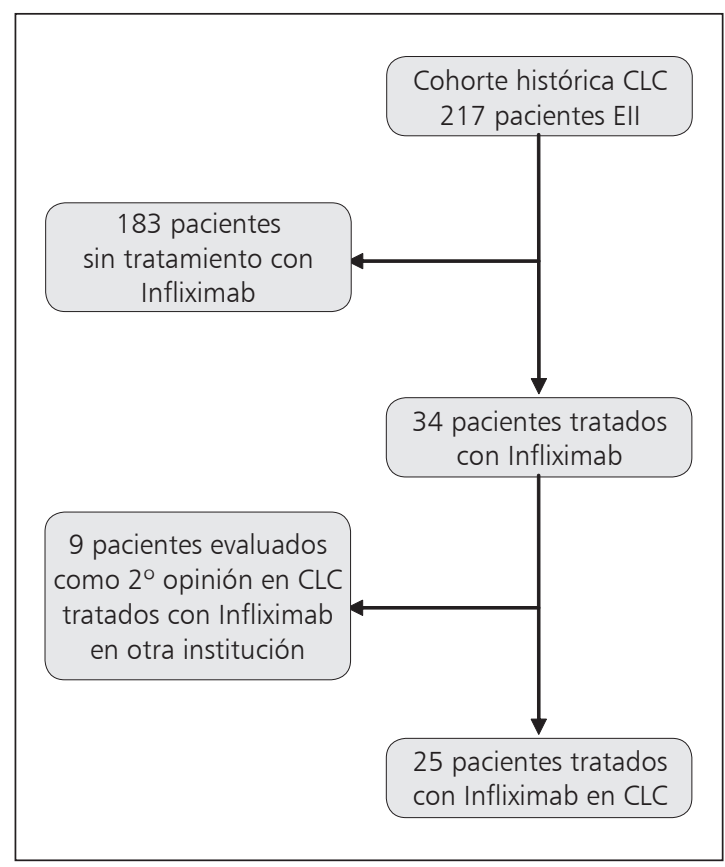

Figura 1. Flujograma selección de pacientes.

según la Clasificación de Montreal para EC y CU. Diez pacientes iniciaron tratamiento dentro de los dos años desde el diagnóstico de su enfermedad. Veintidós pacientes recibieron terapia biasociada, ya sea con azatioprina o metotrexato. El principal motivo de indicación fue el compromiso perianal en EC en 9 pacientes. El número total de dosis de infliximab administradas en todo el grupo fue de 2 a 60. Sólo uno de los 25 pacientes mantenía un hábito tabáquico (Tabla 1).

Veintidós de los 25 pacientes (88\%) presentaron una buena respuesta clínica a infliximab a los 3 a 6 meses de tratamiento, dieciséis de ellos asociado a remisión endoscópica y/o radiológica (64\%). En el grupo de pacientes con CU, ocho de los nueve pacientes respondieron clínicamente a esta terapia. De ellos, cinco presentaron remisión endoscópica con un Mayo 0-1 (Tabla 2). En los tres pacientes que no se observó respuesta endoscópica, sí se evidenció una remisión de las lesiones de un Mayo 3 a un Mayo 2 decidiéndose mantener el tratamiento con infliximab. Hubo un paciente que no respondió ni clínica ni endoscópicamente, quien presentaba una $\mathrm{CU}$ severa refractaria a esteroides e inmunosupresores, con una colonoscopia Mayo 3, realizándose finalmente una colectomía en tres tiempos. En un paciente de este grupo la indicación de infliximab fue por presentar un pioderma gangrenoso como manifestación extraintestinal, recibiendo sólo dos dosis de infliximab debido a una respuesta completa de sus lesiones dermatológicas. Hasta la fecha solamente un paciente con CU ha completado más de un año de tratamiento con infliximab, manteniéndose en remisión clínica.

En el grupo de pacientes con EC, 14 de 16 pacientes $(87 \%)$ presentaron una respuesta clínica favorable posterior a tres dosis de infliximab. En el grupo con fenotipo inflamatorio (cinco pacientes), todos presentaron una buena respuesta clínica y endoscópica a los tres a seis meses. Cuatro de ellos han sido tratados por más de un año con buena respuesta, uno de ellos requirió aumento de la dosis de infliximab a $10 \mathrm{mg} / \mathrm{kg}$ por reactivación, con buena respuesta. De los nueve pacientes con indicación por fístula perianal (todos tratados con seton), ocho presentaron remisión clínica, seis de estos asociados también a remisión radiológica. Hasta la fecha, nueve pacientes con EC han completado un año de tratamiento, ocho de ellos manteniéndose en remisión (Tabla 3). Dos pacientes han requerido cirugía por falla al tratamiento (ileostomía y fistulotomía completa respectivamente). Finalmente, dos pacientes con fenotipo estenosante de íleon, uno de ellos además con fístulas ileocolónicas y el otro con antecedente de resección ileocolónica, no presentaron respuesta ni clínica ni radiológica, motivo por el cual fueron sometidos a resección ileal.

Durante el período de estudio, doce pacientes suspendieron la terapia, tres por mala respuesta, seis por decisión del paciente/médico y tres por costo económico (Tabla 4). Dos de los seis pacientes reiniciaron terapia biológica por reactivación de sus fístulas luego de haber abandonado el tratamiento.

Tres pacientes presentaron eventos adversos (herpes zoster y sinusitis), todos en terapia combinada con azatioprina o metotrexato, ninguno de estos eventos motivó la suspensión del tratamiento.

\section{Discusión}

Este es el segundo estudio nacional que muestra el efecto favorable de infliximab en el 
Infliximab en enfermedad inflamatoria intestinal - D. Simian et al

Tabla 1. Características enfermedad inflamatoria intestinal

\begin{tabular}{|c|c|c|c|}
\hline & $\begin{array}{c}E C \\
n=16\end{array}$ & $\begin{array}{c}\text { CU } \\
n=9\end{array}$ & $\begin{array}{c}\text { Total } \\
n=25\end{array}$ \\
\hline \multicolumn{4}{|l|}{ Sexo } \\
\hline Femenino & 7 & 5 & 12 \\
\hline Masculino & 9 & 4 & 13 \\
\hline Edad (mediana y rango) & $31(18-61)$ & $41(20-54)$ & $36(18-61)$ \\
\hline \multicolumn{4}{|l|}{ Clasificación de Montreal } \\
\hline \multicolumn{4}{|l|}{ Enfermedad de Crohn } \\
\hline \multicolumn{4}{|l|}{ Ubicación } \\
\hline íleon terminal & 3 & & \\
\hline Colon & 6 & & \\
\hline íleon y colon & 7 & & \\
\hline \multicolumn{4}{|l|}{ Fenotipo* } \\
\hline Inflamatorio & 5 & & \\
\hline Estenosante & 2 & & \\
\hline Fistulizante & 1 & & \\
\hline Perianal & 9 & & \\
\hline \multicolumn{4}{|l|}{ Colitis ulcerosa } \\
\hline \multicolumn{4}{|l|}{ Ubicación } \\
\hline Proctitis & & 1 & \\
\hline Colitis izquierda & & 2 & \\
\hline Colitis extensa & & 6 & \\
\hline \multicolumn{4}{|l|}{$\begin{array}{l}\text { Tratamiento asociado al momento } \\
\text { de la terapia biológica\# }\end{array}$} \\
\hline Azatioprina & 12 & 8 & 20 \\
\hline Metotrexato & 1 & 1 & 2 \\
\hline Esteorides & 3 & 3 & 6 \\
\hline Mesalazina & 0 & 5 & 5 \\
\hline Monoterapia & 3 & 0 & 3 \\
\hline \multicolumn{4}{|l|}{ Motivo de indicación } \\
\hline Refractariedad a terapia esferoidal & 0 & 5 & 5 \\
\hline Refractariedad a inmunosupresores & 5 & 2 & 7 \\
\hline Compromiso perianal en EC & 9 & 0 & 9 \\
\hline Estenosis en EC & 2 & 0 & 2 \\
\hline Evento adverso a Mesalazina y AZT & 0 & 1 & 1 \\
\hline Manifestación extraintestinal & 0 & 1 & 1 \\
\hline
\end{tabular}

$\mathrm{EC}=$ Enfermedad de Crohn, CU = Colitis Ulcerosa, AZT = Azatioprina. \#Pacientes pueden tener más de un tratamiento asociado a la terapia biológica. *Un paciente con fenotipo estenosante y fistulizante.

Tabla 2. Respuesta clínica y endoscópica al tratamiento con Infliximab

\begin{tabular}{|c|c|c|c|}
\hline & $\begin{array}{c}E C \\
n=16\end{array}$ & $\begin{array}{c}\text { CU } \\
n=9\end{array}$ & $\begin{array}{c}\text { Total } \\
n=25(\%)\end{array}$ \\
\hline \multicolumn{4}{|l|}{ Respuesta clínica } \\
\hline Buena respuesta & 14 & 8 & $22(88)$ \\
\hline Sin respuesta & 2 & 1 & $3(12)$ \\
\hline \multicolumn{4}{|c|}{ Respuesta endoscópica y/o imagen } \\
\hline Buena respuesta * & 11 & 5 & $16(64)$ \\
\hline Sin respuesta & 5 & 3 & $8(32)$ \\
\hline No evaluado \# & 0 & 1 & $1(4)$ \\
\hline
\end{tabular}

*En CU índice de Mayo 0-1, en EC puntaje $\leq 2$ según score endoscópico SES-CD. \#Paciente con indicación de Infliximab por Pioderma gangrenoso no fue evaluado ya que recibió sólo 2 dosis. 
Tabla 3. Respuesta al tratamiento con Infliximab en pacientes con EC a los 3-6 meses y al año de tratamiento

\begin{tabular}{|lcc|}
\hline Respuesta a los 3-6 meses & $\begin{array}{c}\text { EC Inflamatorio } \\
\text { n: } \mathbf{5}\end{array}$ & $\begin{array}{c}\text { EC Perianal } \\
\text { n: } \mathbf{9}^{\#}\end{array}$ \\
Clínica & 5 & 8 \\
Endoscópica + Clínica & 5 & 6 \\
Radiológica + Clínica & & EC Perianal \\
Respuesta al año & EC Inflamatorio & $\mathbf{n}$ \\
Clínica & $\mathbf{n : ~ 4}$ & 5 \\
Endoscópica y/o Radiológica + Clínica & 4 & 4 \\
\hline
\end{tabular}

\#Todos los pacientes fueron tratados con Seton previo a la terapia con Infliximab.

Tabla 4. Suspensión tratamiento con Infliximab: causa, dosis recibida y respuesta obtenida por paciente

\begin{tabular}{|c|c|c|c|}
\hline Paciente & Causa suspensión & $\begin{array}{l}n \text { dosis } \\
\text { recibidas }\end{array}$ & $\begin{array}{l}\text { Respuesta clínica/endoscópica } \\
\text { obtenida }\end{array}$ \\
\hline $\begin{array}{l}1 \\
2 \\
3 \\
4 \\
5\end{array}$ & $\begin{array}{l}\text { Decisión médico } \\
\text { Indicación pioderma gangrenoso } \\
\text { Buena respuesta } \\
\text { Buena respuesta } \\
\text { Cirugía por subobstrucción intestinal } \\
\text { Cirugía por estenosis }\end{array}$ & $\begin{array}{r}2 \\
5 \\
3 \\
2 \\
60\end{array}$ & $\begin{array}{l}\text { Buena/No evaluada } \\
\text { Buena/Buena } \\
\text { Buena/Buena } \\
\text { Sin respuesta/Sin respuesta } \\
\text { Buena inicial/Buena inicial }\end{array}$ \\
\hline 6 & $\begin{array}{l}\text { Decisión paciente } \\
\text { Paciente discontinúa terapia }\end{array}$ & 16 & Buena/Buena \\
\hline $\begin{array}{l}7 \\
8 \\
9\end{array}$ & $\begin{array}{l}\text { Costo-Económico } \\
\text { Imposibilidad de costear el medicamento } \\
\text { Imposibilidad de costear el medicamento } \\
\text { Imposibilidad de costear el medicamento }\end{array}$ & $\begin{array}{r}3 \\
3 \\
10\end{array}$ & $\begin{array}{l}\text { Buena/No evaluada } \\
\text { Buena/Buena } \\
\text { Buena/Buena }\end{array}$ \\
\hline
\end{tabular}

tratamiento de la EII. A diferencia del estudio de Gellona y cols. ${ }^{24}$, nosotros evaluamos la respuesta posterior a las tres dosis de inducción y en algunos casos la respuesta al año de haber utilizado infliximab. Además, hemos incluido pacientes con CU, grupo en el cual el uso de infliximab ha sido aprobado, ${ }^{9,15}$.

Los resultados obtenidos en nuestro estudio, con $64 \%$ de respuesta endoscópica y/o radiológica son concordantes con cohortes internacionales en relación al uso de infliximab como tratamiento en EII, los cuales muestran respuesta clínica a las 2 y 30 semanas en $58 \%$ y $30 \%$ respectivamente ${ }^{25}$. En los pacientes con EC fenotipo perianal estudios han demostrado reducción $\geq 50 \%$ de las fístulas en $68 \%$ de los pacientes a los tres meses de tratamiento con infliximab ${ }^{26}$, concordante con los resultados obtenidos en nuestro estudio (66\%).
Se ha demostrado el beneficio de mantener la terapia con infliximab en aquellos pacientes que respondieron a las dosis iniciales, mostrando remisión clínica en 30\% de los pacientes al año de tratamiento ${ }^{25}$. En nuestra cohorte, $90 \%$ de los pacientes que completaron un año de tratamiento respondieron clínicamente. Aunque infliximab en estudios poblacionales no ha disminuido la necesidad de cirugía en pacientes con $\mathrm{EC}^{27}$, su rol en la prevención de la recurrencia post operatoria en EC se encuentra avalada por la literatura ${ }^{28}$. Por otra parte, en un período corto de tiempo, la disponibilidad y uso de los anti-TNF $\alpha$ ha ayudado a cambiar el algoritmo terapéutico de los pacientes con CU. Su uso ha demostrado eficacia en lograr remisión libre de esteroides, curación de la mucosa y cambiar la historia natural de la enfermedad (colectomías) $)^{15,29}$. Además, en pacientes con CU 
moderada a severa tratados con este fármaco se ha visto un riesgo significativamente menor de colectomía a las 54 semanas de tratamiento, comparado con placebo ${ }^{4}$.

Es importante evaluar las indicaciones para iniciar la terapia con infliximab en estos pacientes. La indicación principal es refractariedad a las terapias convencionales ${ }^{9,12-14}$. En nuestro estudio esta causa fue $48 \%$, seguido por compromiso perianal en EC (36\%). Se indicó la terapia en dos pacientes con EC estenosante, en los cuales se intentó disminuir el componente inflamatorio de la estenosis, no obteniéndose buenos resultados. Infliximab también ha demostrado ser efectivo en pacientes con CU severa refractaria a esteroides ${ }^{29}$. En nuestro estudio, un solo paciente con CU refractaria requirió cirugía antes de los tres meses, confirmando la efectividad de esta terapia en este grupo de pacientes.

Dentro de las manifestaciones extraintestinales, el pioderma gangrenoso aparece en 1-5\% de los pacientes con EII, siendo más frecuente en pacientes con CU. El uso de infliximab ha sido descrito en pacientes con pioderma gangrenoso, sin embargo, el esquema de administración ha sido variable $^{30}$. Similar a lo descrito por otros autores, la respuesta en nuestro paciente con $\mathrm{CU}$ y pioderma gangrenoso fue completa ${ }^{31,32}$.

Estudios han sugerido que el uso precoz de infliximab (top-down), se ha relacionado con un mejor control de la inflamación, lo que se traduce en una disminución de las secuelas crónicas, como estenosis y fístulas complejas entre otros, que implican la necesidad de cirugía en los pacientes con EII ${ }^{33-35}$. El estudio SONIC demostró que en pacientes con EC vírgenes a inmunosupresores el uso combinado de infliximab y azatioprina es más efectivo en lograr la remisión libre de esteroides y la curación de la mucosa en comparación con ambos grupos de fármacos de manera separada ${ }^{33}$. Sin embargo, otros han puesto en discusión el uso indiscriminado de una estrategia top-down sugiriendo que una estrategia step-up acelerada parece más razonable $e^{36,37}$. Aunque en nuestro estudio, la mayoría de los pacientes (60\%) inició tratamiento con infliximab después de los 2 años del diagnóstico, el porcentaje de efectividad a este fármaco confirma la necesidad de optimizar la terapia tradicional en términos de identificar mejor la dependencia a esteroides, el uso precoz de inmunomoduladores y reevaluar el momento de la cirugía. Por otra parte, es importante determinar la presencia de factores predictores de mal pronóstico al momento de iniciar la terapia biológica de manera precoz. Estudios han determinado que ciertos grupos de pacientes con EII pueden beneficiarse de esta estrategia, destacando aquellos que presenten índices de actividad elevados, edad joven al diagnóstico, hábito tabáquico en pacientes con EC, fenotipo penetrante, compromiso intestinal difuso, úlceras profundas en la endoscopia, compromiso perianal y más de una manifestación extraintestinal ${ }^{36}$.

Durante el tratamiento con infliximab los pacientes pueden presentar una pérdida de respuesta. Dentro de las estrategias a usar cuando esto ocurre está aumentar la dosis de infliximab, acortar los intervalos entre las infusiones (cada seis semanas) o cambiar de terapia biológica ${ }^{38}$, siendo más eficaz la primera apuesta ${ }^{39}$. Estudios han demostrado que la evaluación de los niveles de infliximab y la presencia de anticuerpos contra infliximab permitirían definir la mejor estrategia ${ }^{40}$. En nuestro estudio se aumentó la dosis de infliximab a $10 \mathrm{mg} / \mathrm{kg}$ en tres pacientes, logrando mantener la remisión.

Dentro de los eventos adversos se ha descrito un aumento en el riesgo de linfoma en EC en asociación a inmunomoduladores ${ }^{12,13}$. En pacientes jóvenes en terapia asociada a inmunosupresores aumenta el riesgo de linfoma hepatoesplénico de células $\mathrm{T}^{12}$. Además, estudios han señalado mayor riesgo de infecciones oportunistas ${ }^{2,12,13}$, micóticas y de reactivación de tuberculosis ${ }^{2,8,41}$, principalmente con su uso asociado a esteroides e inmunomoduladores ${ }^{8,14,41}$. En nuestro estudio sólo tres pacientes presentaron infecciones, las cuales no fueron graves ni motivaron la suspensión de la terapia. Ninguno de nuestros pacientes presentó reacciones adversas relacionadas con la infusión de infliximab. Un paciente completó 10 años de tratamiento con infliximab sin presentar eventos adversos asociados a su uso.

Nuestro estudio tiene algunas limitaciones. El tamaño de la muestra es pequeño y es una experiencia local de nuestra institución. Se habría deseado comparar la respuesta clínica y endoscópica al tratamiento con infliximab entre el grupo con EC y CU, pero debido al tamaño de la muestra, ello podría dar resultados sin significancia estadística. Es necesario realizar estudios multicéntricos, prospectivos, para mejorar el grado de evidencia a nivel nacional sobre la eficacia del uso de esta 
terapia en pacientes con EII, así como el riesgo de eventos adversos en un seguimiento a largo plazo.

En conclusión, la introducción de infliximab en el manejo de los pacientes con EII tiene un efecto importante en la evolución de su enfermedad. El uso prolongado de esta estrategia parece ser seguro, además de mantener su efectividad. Es necesario definir el grado de discapacidad de los pacientes tratados para poder determinar el impacto del tratamiento en el curso natural de la enfermedad.

Agradecimientos: Agradecemos a la E.U. Magdalena Castro, MSc@ Epidemiología, de la Subdirección de Investigación, Clínica Las Condes, por su ayuda en el análisis estadístico.

\section{Referencias}

1. Feagan B, Lémann M, Befrits R, Connell W, D’Haens G, Ghosh S. Recommendations for the Treatment of Crohn's Disease with Tumor Necrosis Factor Antagonists: An Expert Consensus Report. Inflamm Bowel Dis 2012; 18: 152-60.

2. Rogler G, Bernstein C, Sood A, Lee Goh K, YamamotoFurusho J, Abbas Z. Role of biological therapy for inflammatory bowel disease in developing countries. Gut 2012; 61: 706-12.

3. Triantafillidis J, Merikas E, Georgopoulos F. Current and emerging drugs for the treatment of inflammatory bowel disease. Drug Design, Development and Therapy 2011; 5: 185-210.

4. Sandbor WJ, Rutgeerts P, Feagan BG, Reinisch W, Olson A, Johanns J, et al. Colectomy rate comparison alter treatment of ulcerative colitis with placebo or infliximab. Gastroenterology 2009; 137: 1250-60.

5. Dignass A, Van Assche G, Lindsay JO, Lémann M, Söderholm J, Colombel JF. The second European evidencebased Consensus on the diagnosis and management of Crohn's disease: Current management. J Crohns Colitis 2010; 4: 28-62.

6. Dignass A, Lindsay JO, Sturm A, Windsor A, Colombel JF, Allez M. Second Erupean evidence-based consensus on the diagnosis and management of ulcerative colitis Part 2: Current management. J Crohns Colitis 2012; 6: 991-1030.

7. Talley N, Abreu M, Achkar J-P, Bernstein C, Dubinsky M, Hanauer S. An evidence-based systematic review on medical therapies for inflammatory bowel disease. Am J Gastroenterol 2011; 106: S2-S25.

8. Hoentjen F, van Bodegraven A. Safety of anti-tumor necrosis factor therapy in inflammatory bowel disease. World J Gastroenterol 2009; 15: 2067-73.

9. Quera R. Aplicación práctica del uso de la terapia antiTNF en la enfermedad de Crohn: ¿Cuándo comenzar, cuál elegir, cómo predecir la respuesta, cuándo cambiar y cuándo suspender? Gastroenterol Latinoam 2012; 23: 49-53.

10. Sandborn WJ, Feagan BG, Marano C, Zhang H, Strauss $\mathrm{R}$, Johanns J, et al. Subcutaneous Golimumab Induces Clinical Response and Remission in Patients with Moderate-To-Severe Ulcerative Colitis. Gastroenterology 2013 [Epub ahead of print].

11. Bosani M, Ardizzone S, Bianchi Porro G. Biologic targeting in the treatment of inflammatory bowel diseases. Biologics: Targets \& Therapy 2009; 3: 77-97.

12. Orlando A, Armuzzi A, Papi C, Annese V, Ardizzone S, Biancone L. The Italian Society Gastroenterology (SIGE) and the Italian Group for the study of Inflammatory Bowel Disease (IG-IBD) Clinical Practice Guidelines: The use of the tumor necrosis factor-alpha antagonist therapy in Inflammatory Bowel Disease. Digestive and Liver Disease 2010; 43: 1-20.

13. Ordás I, Feagan B, Sandborn W. Early use of inmmunosuppressives or TNF antagonists for the treatment of Crohn's disease: time for a change. Gut 2011; 60: 175463.

14. D'Haens G, Panaccione R, Higgins P, Vermeire S, Gassull M, Chowers Y. The London Position Statement of the World Congress of Gastroenterology on Biological Therapy for IBD With the European Crohn's and Colitis Organisation: When to Start, When to Stop, Which Drug to Choose, and How to Predict Response? Am J Gastroenterol 2011; 106: 199-212.

15. Danese S, Colombel JF, Peyrin-Biroulet L, Rutgeerts P, Reinisch W. Review article: the role of anti-TNF in the management of ulcerative colitis-past, present and future. Aliment Pharmacol Ther 2013; 37: 855-66.

16. Ahluwalia J. Immunotherapy in inflammatory bowel disease. Med Clin N Am 2012; 96: 525-44.

17. Pavez C, Ruiz M, Bretón I, Palma S, Del Barrio P, Candia R. Terapia para la enfermedad de Crohn: infliximab, azatioprina o combinación. Gastroenterol Latinoam 2011; 22: $277-80$.

18. Van Assche G, Dignass A, Panes J, Beaugerie L, Karagiannis J, Allez M, et al; for the European Crohn's and Colitis Organisation (ECCO). The second European evidencebased Consensus on the diagnosis and management of Crohn's disease: Definitions and diagnosis. J Crohns Colitis 2010; 4: 7-27.

19. Dignass A, Eliakim R, Magro F, Maaser C, Chowers Y, Geboes K. Second European evidence-based consensus 
on the diagnosis and management of ulcerative colitis Part 1: Definitions and diagnosis. J Crohns Colitis 2012; 6: 965-90.

20. Lewis J, Chuai S, Nessel L, Lichtenstein G, Aberra F, Ellenberg J. Use of the noninvasive components of the Mayo Score to assess clinical response in ulcerative colitis. Inflamm Bowel Dis 2008; 14: 1660-6.

21. af Björkesten CG, Nieminen U, Turunen U, Arkkila P, Sipponen T, Färkkilä M. Surrogate markers and clinical indices, alone or combines, as indicators for endoscopic remission in anti-TNF-treated luminal Crohn's disease. Scand J Gastroenterol 2012; 47: 528-37.

22. Daperno M, D’Haens G, Van Assche G, Baert F, Bulois P, Maunoury V, et al. Development and validation of a new, simplified endoscopic activity score for Crohn's disease: the SES-CD. Gastrointest Endosc 2004; 60: 50512 .

23. Tozer P, Ng SC, Siddiqui MR, Plamondon S, Burling D, Gupta A, et al. Long-term MRI-guided combined antiTNF- $\alpha$ and thiopurine therapy for Crohn's perianal fistulas. Inflamm Bowel Dis 2012; 18: 1825-34.

24. Gellona J, Zarraonandia A, Zúñiga A, Palma R, Contreras J, Silva J, et al. Infliximab en el tratamiento de la Enfermedad de Crohn. Estudio preliminar. Rev Med Chile 2006; 134: 320-5.

25. Hanauer SB, Feagan BG, Lichtenstein GR, Mayer LF, Schreiber S, Colombel JF, et al. Maintenance infliximab for Crohn's disease: the ACCENT I randomised trial. Lancet 2002; 359: 1541-9.

26. Present DH, Rutgeerts P, Targan S, Hanauer SB, Mayer L, van Hogezan RA, et al. Infliximab for the treatment of fistulas in patients with Crohn's disease. N Engl J Med 1999; 340: 1398-405.

27. Latella G. Surgery for Crohn's disease in the era of biologicals: A reduced need or delayed verdict? World J Gastroenterol 2012; 18: 3828-32.

28. Yamamoto T. Prevention of recurrence after surgery for Crohn's disease: Efficacy of Infliximab. World J Gastroenterol 2010; 16: 5405-10.

29. Laharie D, Bourreille A, Branche J, Allez M, Bouhnik Y, Filippi J. Ciclosporin versus infliximab in patients with severe ulcerative colitis refractory to intravenous steroids: a parallel, open-label randomised controlled trial. Lancet 2012; 380: 1909-15.

30. Carrasco C, Ruiz MM, Salaberri JJ, Pérez JJ. Pyoderma gangrenosum associated with inflammatory bowel disease. Report of two cases with good response to infliximab. Reumatol Clinic 2012; 8: 90-2.
31. Sapienza MS, Cohen S, Dimarino AJ. Treatment of pyoderma gangrenosum with infliximab in Crohn's disease. Dig Dis Sci 2004; 49: 1454-7.

32. Brooklyn TN, Dunnill MG, Shetty A, Bowden JJ, Williams JD, Griffiths CE, Forbes A, Greenwood R, Probert CS. Infliximab for the treatment of pyoderma gangrenosum: a randomised, double blind, placebo controlled trial. Gut 2006; 55: 505-9.

33. Colombel JF, Sandborn WJ, Reinisch W, Mantzaris GJ, Kornbluth A, Rachmilewitz D, et al. Infliximab, azathioprine, or combination therapy for Crohn's disease. N Engl J Med 2010; 362: 1368-95.

34. Hommes DW, Baert F, van Assche G, Caenepeel F, Vergauwe $\mathrm{P}$, Tuynman $\mathrm{H}$, et al. Therapy management of recent onset Crohn's disease: A controlled, randomized trial comparing step-up and top-down therapy. Gastroenterology 2005; 129: 371.

35. D’Haens G, Baert F, van Assche G, Caenepeel P, Vergauwe P, Tuynman H, et al. Early combined immunosuppression or conventional management in patients with newly diagnosed Crohn's disease: an open randomised trial. Lancet 2008; 371: 660-7.

36. Ghazi L, Patil S, Rustgi A, Flasar M, Razeghi S and Cross R. Step up versus early biologic therapy for crohn's disease in clinical practice. Inflammatory Bowel Dis 2013; 19: 1397-403.

37. Fascì Spurio F, Aratari A, Margagnoni G, Doddato MT, Papi C. Early treatment in Crohn's disease: do we have enough evidence to reverse the therapeutic pyramid? J Gastrointestin Liver Dis 2012; 21: 67-73.

38. Danese S, Fiorino G, Reinisch W. Review article: causative factors and the clinical management of patients with Crohn's disease who lose response to anti-TNF- $\alpha$ therapy. Aliment Pharmacol Ther 2011; 34: 1-10.

39. Katz L, Gisbert JP, Manoogian B, Lin K, Steenholdt C, Mantzaris GJ. Doubling de infliximab dose versus halving the infusión intervals in Crohn's disease patients with loss of response. Inflamm Bowel Dis 2012; 18: 2026-33.

40. Eser A, Primas C, Reinisch W. Curr Opin Gastroenterol. Drug monitoring of biologics in inflammatory bowel disease. Curr Opin Gastroenterol 2013; 29: 391-6.

41. Van Assche G, Lewis J, Lichtenstein G, Loftus E, Ouyang Q, Panes J. The London Position Statement of the World Congress of Gastroenterology on Biological Therapy for IBD with the European Crohn's and Colitis Organisation: Safety. Am J Gastroenterol 2011; 106: 1594-602. 